\title{
UNSUAL BEHAVIOR OF COLLOID BREAST CARCINOMA: CASE REPORT
}

Anna Carolina Pereira Jacome ${ }^{1}$, Alice Morais de Castro Silva ${ }^{1}$, Daniela Beggiato Correa ${ }^{1}$, Ingrid Bernucci Netoํ, Patrícia Aguiar Bellini ${ }^{1}$

${ }^{1}$ Hospital Felício Rocho - Belo Horizonte (MG), Brazil

Introduction : Colloid breast carcinoma is a rare variant of invasive ductal carcinoma, representing $1 \%$ to $6 \%$ of the cases. It is characterized by rich deposition of extracellular mucin upon anatomopathological analysis (AP). In general, it has positive hormone receptors, inclusion in the Luminal A molecular subtype, and an epidemiological profile of older women. Its behavior is usually indolent, associated with a low grade and favourable prognosis. It is unlikely to infiltrate axillary lymph nodes or be associated with metastatic potential. We present a case report of a patient with invasive colloid carcinoma of the breast associated with ductal carcinoma in situ (DCIS) and early axillary recurrence of a colloid pattern, contesting the mild behavior that is the gold standard in the literature. Case report: A 63-year-old patient, without relevant risk factors, was admitted due to a palpable nodule in the upper outer quadrant (UOQ) of her left breast (LB). Radiological exams showed a nodular lesion of 15x16x10 mm, heterogeneous and non-circumscribed associated with microcalcifications, with core biopsy showing colloid carcinoma. The axillae were clinically and radiologically negative. She underwent a sentinel lymph node biopsy and lumpectomy. AP results showed invasive colloid carcinoma, grade 2, measuring $1.7 \mathrm{~cm}$, associated with cribiform DCIS occupying $20 \%$ of the total area of the neoplasia, free margins and three negative sentinel lymph nodes. Immunohistochemistry was compatible with Luminal A. Treatment with radiotherapy and the use of tamoxifen followed. During follow-up, in July 2020, a physical examination showed thickening in the UOQ and a hardened palpable lymph node on the left. Routine examinations showed a complex nodule compatible with clinical thickening in the UOQ and grouped and heterogeneous microcalcifications in the retroareollar region of the LB, in addition to axillary lymphadenopathy with asymmetric cortical thickening at ipsilateral level I. For better elucidation, breast magnetic resonance imaging was requested, which showed areas suggestive of fibrocicatrial changes and suspicious-looking lymph node enlargement on the left. Fine-needle aspiration biopsy (FNAB) guided by ultrasound was performed, compatible with positivity for atypical cells. Thereby, a new surgical approach was performed: mastectomy and level I axillary node dissection on the left. AP was compatible with $0.6 \mathrm{~mm}$ DCIS associated with intraductal papilloma without atypia of LB and 1 in 6 lymph nodes, positive for diffuse infiltration by colloid pattern carcinoma. Today, the patient is being followed up, using anastrozole, without oncological changes in other follow-up exams. Cases that deviate from the standard in literature, such as the exposed surprisingly aggressive behavior of a rare variant, should be reported to encourage discussions about adequate approach and treatment. 